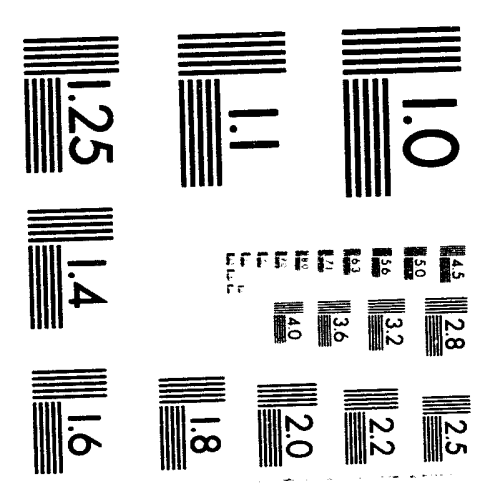



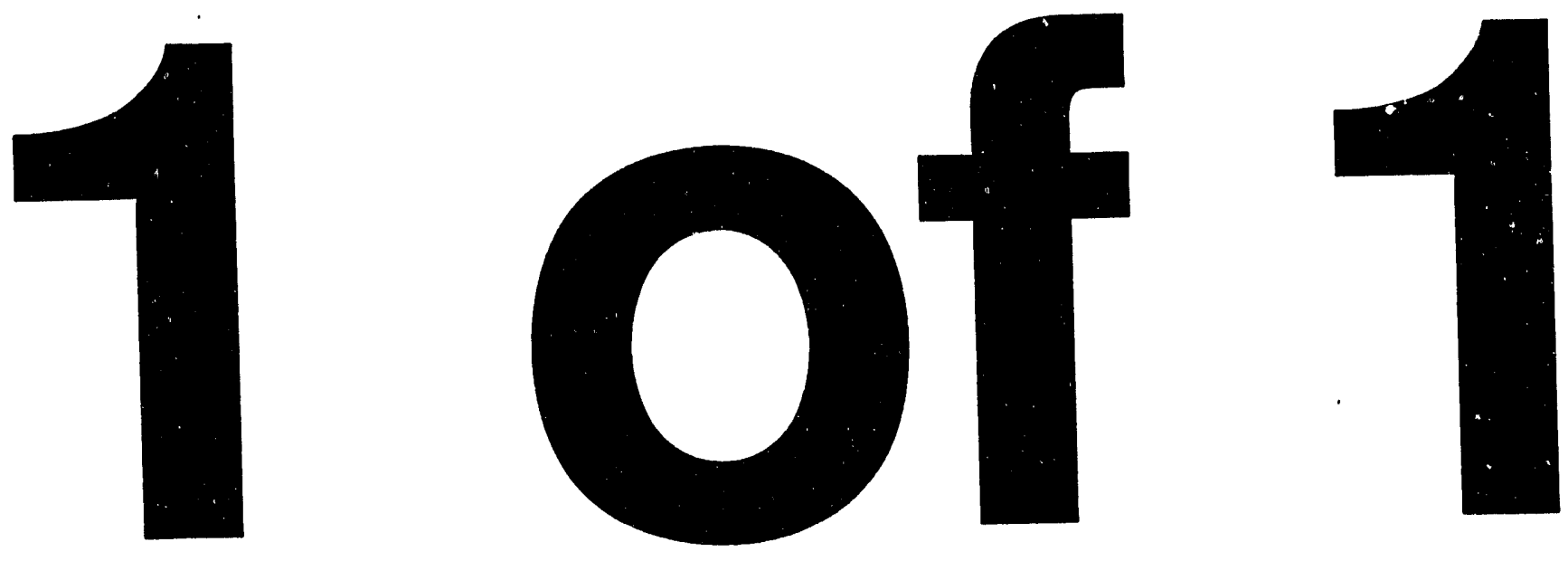


\section{A Comparison Between Two Monte Carlo Codes on Determination of Transient Chemical Yields}

\section{R. N. Hamm, A. Chatterjee ${ }^{b}$ and J. E. Turner}

Abstract

Monte Carlo computer codes have been independently developed at several laboratories for performing calculations of the radiolysis of water. The different codes involve a wide variety of models and related assumptions in treating the many physical and chemical processes that occur. Because few detailed aspects of such computations can be directly checked by experiment, it is important to make comparisons of various predicted microscopic distributions. In this paper we compare results obtained with the codes developed at Lawrence Berkeley Laboratory and at Oak Ridge National Laboratory. Both codes were used to calculate the spatial distributions of various radical species in spurs along the tracks of energetic electrons. Similarities and differences in the results of this preliminary study are shown. Additional work is planned.

\section{Introduction}

Radiation-induced damage to a living cell begins with the physical stage of energy deposition on the various target molecules present in the system. Among the variety of target molecules with which radiation can interact, water molecules and DNA are considered to be critically important ${ }^{(1)}$. Both of these molecules can undergo the primary processes of ionization and excitation, which are generally complete in about $10^{-15} \mathrm{~s}$. However, the damage to DNA (e.g., strand breaks, base alterations, etc.) from these fast processes can evolve over several orders of magnitude in time, but the kinetics associated with them have not been studied in great detail. On the contrary, a time-dependent analysis of the ensuing chemical stage that follows after the absorption of energy by water molecules has provided us with a better understanding of the time evolution of water-radical-induced DNA damage. Most of these studies are theoretical in nature, and it has been increasingly clear that experimental techniques are not likely to improve enough to allow exploration of the time interval between $10^{-15} \mathrm{~s}$ and $10^{-12} \mathrm{~s}$. Following the absorption of energy by water molecules, new chemical species such as hydroxyl radicals, hydrogen radicals, solvated electrons, etc., are produced within the cellular complex. These species become thermalized in about $10^{-12} \mathrm{~s}$. At this point in the time scale, diffusion of radicals begins and various

\footnotetext{
Research sponsored by the Office of Health and Environmental Research, U.S. Department of Energy, under contract DE-AC05-84OR21400 with Martin Marietta Energy Systems, Inc. and under contract DE-AC03-76SF00098 with the University of California.
}

${ }^{\star H}$ Health Sciences Research Division. Oak Ridge National Laboratory, Oak Ridge, TN 37831-6123

${ }^{b}$ Life Sciences Division, Lawrence Berkeley Laboratory, Berkeley, CA 94720 
chemical reactions follow, some of which result in DNA damage. Water radicals can react with each other and produce molecular products, such as $\mathrm{H}_{2}, \mathrm{H}_{2} \mathrm{O}_{2}, \mathrm{H}_{2} \mathrm{O}$, etc. Radicals which escape such reaction can lead to DNA damage. Hence it is extremely important to evaluate quantitatively the yields of various radical species as functions of time including information on spatial distributions.

There are several theoretical approaches which deal with the calculation of yields of chemical species produced as a result of radiolysis of water. For example, Zaider et al. ${ }^{(2)}$ have developed a Monte Carlo scheme using gas-phase cross sections for electronic energy loss. Then they extrapolate to unit density for applicability in the liquid phase. Their strategy is based on the fact that much more extensive experimental data are available for the vapor phase than for the liquid phase. It is not clear whether this procedure adequately describes the liquid-phase cross sections. However, their time-dependent decay curves for $\mathrm{OH}$ as well as $e_{2 q}$ have similar shapes to those observed experimentally by Jonah et al. ${ }^{(3,4)}$ In a different approach, Brenner and Zaider ${ }^{(s)}$ use Monte Carlo methods to obtain the spatial distributions of water radicals at $\sim 10^{-12} \mathrm{~s}$, which become the input to another code, based on Smoluchowski's equation, that crimputes events to later times. In contrast to these approaches, Chatterjee and Magee ${ }^{(6)}$ initiate the computation with an assumed Gaussian distribution following the passage of a charged particie. In their method, they utilize track entities called "spurs", "blobs", and "short tracks" for electron iriadiation and "core" and "penumbra" for heavy-charged particles. This approach has now been extended to evaluate the yields of DNA strand breaks. However, in the Chatterjee and Magee model developed at the Lawrence Berkeley Laboratory (LBL), the stage between $10^{-15} \mathrm{~s}$ and $10^{-12} \mathrm{~s}$ is treated very approximately with several assumptions. A local energy deposit between $6 \mathrm{eV}$ and $100 \mathrm{eV}$ in water is called a spur; an energy deposit between $100 \mathrm{eV}$ and $500 \mathrm{eV}$ is called a blob; and for short tracks these values lie between $500 \mathrm{eV}$ and $5000 \mathrm{eV}$.

Perhaps the most complete model available at present is the one developed at the Oak Ridge National Laboratory ${ }^{(7,8)}$. In this model, attempts are made to account for every ionization or excitation event in a statistical manner that follows through to the essential completion of intratrack radical reactions at about $10^{-6} \mathrm{~s}$. In liquid water, the initial physical processes are represented by writing

$$
\mathrm{H}_{2} \mathrm{O} \rightarrow \mathrm{H}_{2} \mathrm{O}^{+}, \mathrm{H}_{2} \mathrm{O}^{*}, \mathrm{e}^{-}
$$

The Monte Carlo computer code, OREC, was developed at ORNL to perform the detailed 
transport and energy-loss calculation of the primary charged particle and all of its secondary electrons. For a primary or secondary particle of given energy, the code selects a flight distance to the next event, the type of event that occurs, the energy loss, the scattering angle, and the energy and angle of the secondary electron when an ionization occurs. The inelastic cross sections are derived from a complex dielectric response function developed specifically for liquid water. ${ }^{(9)}$ OREC explicitly considers the physico-chemical and chemical stages. The theoretical decay curves obtained for $\mathrm{OH}$ and $\mathrm{e}_{2 \mathrm{q}}$ are in good agreement with the experimental data of Jonah et al., ${ }^{(3.4)}$ measured between $3 \times 10^{-11} \mathrm{~s}$ and $4 \times 10^{-8} \mathrm{~s}$. In addition, the computed ferric yield for tritium beta rays agrees with experiment.

In view of the several theoretical techniques available, one of the objectives of this conference has been to make a comparison between various methods. This chapter provides a quantitative comparison between various results obtained from the OREC and LBL codes.

In the LBL code, the prethermalization stage is approximated by assuming that the radicals are distributed according to a Gaussian function with a characteristic standard deviation $\sigma$ for each radical. These $\sigma$ values have been adjusted to obtain the best fit of experimental data on steady-state yields of radicals. Conversion of the energy absorption to production of chemical species is accounted for by assuming that $17 \mathrm{eV}$ is required on an average to create a water-radical pair. Based on the $\sigma$ values and the number of radical pairs produced in a spur, random numbers are used to select from a threedimensional Gaussian distribution, and the various chemical species are positioned in space accordingly. Subsequent to this procedure, the next steps in the overall Monte Carlo scheme are very similar to those of the Oak Ridge code (OREC). Since OREC is capable of localizing the initial chemical species without making any assumptions such as prescribed Gaussian functions, it is extremely important to compare the various characteristics of these distributions by the two codes.

The two main characteristics chosen for the present comparison are the spur-size distribution and the various $\sigma$ values. As mentioned earlier, spurs are created by the energy losses below $100 \mathrm{eV}$. Hence, the number of ion pairs (approximately same as radical pairs) will depend upon the energy in a spur. Mozumder and Magee (10) have computed the spur size and its corresponding probability. These can also be computed by the Oak Ridge code and hence form the basis for comparison.

\section{DISCLAIMER}

\footnotetext{
This report was prepared as an account of work sponsored by an agency of the United States Government. Neither the United States Government nor any agency thereof, nor any of their employees, makes any warranty, express or implied, or assumes any legal liability or responsibility for the accuracy, completeness, or usefulness of any information, apparatus, product, or process disclosed, or represents that its use would not infringe privately owned rights. Reference herein to any specific commercial product, process, or service by trade name, trademark, manufacturer, or otherwise does not necessarily constitute or imply its endorsement, recommendation, or favoring by the United States Government or any agency thereof. The views and opinions of authors expressed herein do not necessarily state or reflect those of the United States Government or any agency thereof.
} 


\section{Comparison of Results}

Calculations were made using OREC for segments of $1-\mathrm{MeV}$ electron tracks. In these calculations each energy-loss event by the primary electron was treated independently and categorized as a spur, blob or short track according to the criteria of Chatterjee and Magee. The average yields of the various radicals and their spatial distributions were then tabulated for the different categories from a large number of trials and compared with the corresponding distributions from the LBL model.

Figure 1 shows the probability density $P(x)$ for the location of an $\mathrm{OH}$ radical at a perpendicular distance $x$ from the path of a primary charged particle when the number of radical pairs in a spur is $n=1$. The open circles give the distribution computed from OREC, and the dashed curve (LBL) shows the Gaussia: distribution used by Chatterjee and Magee. ${ }^{(1)}$ The solid curve (ORNL) represents a Gaussian distribution, having the same standard deviation as that of the open circles. The dashed and solid curves virtually coincide. All distributions are normalized, both here and in the following figures.

Figure 2 preseits the distribution of $\mathrm{OH}$-radical positions when there are $n=2$ radical pairs in the spur. The calculated ORNL distribution (open circles) is more widely spread than that of LBL (dashed curve). When a normal distribution is plotted with the same standard deviation as that of the open circles, the solid curve (ORNL) results. The OREC distribution is sharper than Gaussian near the trajectory of the primary particle and extends to larger values of $x$, where the probability density is small.

Figure 3 shows the distribution for the hydrated electron for spurs in which there is only a single radical pair. The Gaussian LBL (dashed curve) and OREC (open circle) distributions are markedly different. When a normal curve is assumed with the same standard deviation as that of the open circles, the solid curve is obtained. Figure 4 presents the saine information for the hydrated electron in spurs where two radical pairs are formed. It is apparent that the OREC model gives much greater displacements in the initial $\left(\sim 10^{-12} \mathrm{~s}\right)$ positions of the electrons from the path of the primary particle than the LBL model. Furthermore, the OREC distribution is only approximately Gaussian.

The three distributions for the hydronium ion for spurs with two radical pairs are plotted in Fig. 5. Here the OREC distributions are tighter than that in the LBL model. The OREC distribution (circles) is somewhat more peaked than the Gaussian curve with the same standard deviation. 
Table 1 summarizes the standard deviations of the radical distributions computed with the LBL and OREC models. $P(n)$ gives the probability that a spur has exactly $n$ radical pairs. The standard deviations for $\mathrm{OH}$ and $\mathrm{H}$ are shown for the two models. In the LBL calculations, the standard deviations for $\mathrm{e}_{2 \mathrm{q}}$ and $\mathrm{H}_{3}{ }^{+} \mathrm{O}$ are shown in the last column of the table. They are both different from that for $\mathrm{H}$ in the OREC model.

Some of the distributions shown here are quite similar for the two models, but others differ greatly. Despite this, both models give good agreement with the measured decay curves for $\mathrm{OH}$ radicals and for hydrated electrons ${ }^{(3,4)}$. Further work is underway to attempt to understand this and to delineate which differences in these distributions are most significant for the final calculated yields. 


\section{Figure Captions}

Figure 1. Distribution of displacements $\mathrm{x}$ of $\mathrm{OH}$ radicals from the path of a primary charged particle in spurs that contain exactly $n=1$ radical pair. The dashed curve represents the LBL Gaussian distribution, the circles show the distribution calculated with OREC, and the solid curve gives a Gaussian curve having the same standard deviation as the OREC points.

Figure 2. Same as Fig. 1 for spurs with exactly $n=2$ radical pairs.

Figure 3. Distributions (same notation as in Figs. 1 and 2) for hydrated electrons with $n=1$.

Figure 4. Same as Fig. 3 for $n=2$.

Figure 5. Distributions for $\mathrm{H}_{3} \mathrm{O}^{+}$with $n=2$ (same notation as before). 


\section{References}

1. J. B. Little, J. N. Whaley and H. L. Liber. Role of Energy Distribution in DNA on the Mutagenic Effects of Internal Emitters. In Mechanisms of Anti-Carcinogenesis and Radiation Protection, O. F. Nygaard and A. C. Upton (eds.), p. 201. Plenum Press, New York (1991).

2. M. Zaider, D. J. Brenner, and W. E. Wilson. The Application of Track Calculations to Radiobiology. Radiat. Res. 95:231-247 (1983).

3. C. D. Jonah and J. R. Miller. Yield and Decay of the OH Radical from 200 ps to 3 ns. J. Phys. Chem. 81:1974-1976 (1977).

4. C. D. Jonah, M. S. Matheson, J. R. Miller, and E. J. Hart. Yield and Decay of the Hydrated Electron from $100 \mathrm{ps}$ to 3 ns. J. Phys. Chem. 80:1267-1270 (1976).

5. D. J. Brenner and M. Zaider. Stochastic and Deterministic Treatments of the Time Decay of Species Created by Heavy-Charged Particle Interactions. Radiat. Prot. Dosimetry 13:127-132 (1985).

6. A. Chatterjee and J. L. Magee. Radiation Chemistry of Heavy Particle Tracks. 2. Fricke Dosimeter System. J. Phys. Chem. 84:3537-3543 (1980).

7. I. E. Turner, J. L. Magee, H. A. Wright, A. Chatterjee, R. N. Hamm, and R. H. Ritchie. Physical and Chemical Development of Electron Tracks in Liquid Water. Radiat. Res. 96:437449 (1983).

8. J. E. Turner. R. N. Hamm, R. H. Ritchie, and Wesley E. Bolch. Monte Carlo Track-Structure Calculations for Aqueous Solutions Containing Biomolecules. Paper in this volume.

9. R. H. Ritchie, R. N. Hamm, J. E. Turner, H. A. Wright, and W. E. Bolch. Radiation Interactions and Energy Transport in the Condensed Phases. In Physical and Chemical Mechanisms in Molecular Radiation Biology, William A. Glass and Matesh N. Varma (eds.), p. 
99. Plenum Press, New York (1991).

10. A. Mozumder and J. L. Magee. The Early Events of Radiation Chemistry. Int. J. Radiat. Phys. Chem. 7:83 (1975).

11. A. Chatterjee and J. L. Magee. Theoretical Investigation of the Production of Strand Breaks in DNA by Water Radicals. Radiat. Prot. Dosimetry 13: 137-140 (1985). 
Table I.

SPUR SIZE DISTRIBUTIONS AND

STANDARD DEVIATIONS FOR RADICALS

\begin{tabular}{||c|c|c|c|c|c|c|c|c||}
\hline \multirow{2}{*}{$n$} & \multicolumn{2}{|c|}{$\mathrm{P}(\mathrm{n})$} & \multicolumn{2}{|c|}{$\sigma_{\mathrm{OH}}(\AA)$} & \multicolumn{2}{c|}{$\sigma_{\mathrm{H}}(\AA)$} & $\sigma_{\text {caa }}$ & $\sigma_{\mathrm{H} 30+}$ \\
\cline { 2 - 9 } & $\mathrm{LBL}$ & ORNL & LBL & ORNL & LBL & ORNL & ORNL & ORNL \\
\hline \hline 1 & 0.48 & 0.304 & 7.5 & 7.4 & 30.0 & 4.2 & 92.1 & 7.5 \\
\hline 2 & 0.35 & 0.377 & 9.5 & 14.2 & 27.7 & 18.6 & 74.6 & 12.0 \\
\hline 3 & 0.10 & 0.165 & 10.8 & 16.6 & 25.5 & 20.6 & 76.8 & 14.2 \\
\hline 4 & 0.04 & 0.074 & 11.9 & 17.3 & 23.5 & 21.7 & 75.4 & 14.9 \\
\hline 5 & 0.02 & 0.039 & 12.8 & 17.2 & 21.7 & 21.4 & 78.1 & 14.8 \\
\hline 6 & 0.01 & 0.024 & 13.6 & 19.1 & 20.0 & 23.7 & 79.0 & 16.6 \\
\hline 7 & & 0.012 & & 18.8 & & 23.1 & 77.5 & 16.2 \\
\hline 8 & & 0.004 & & 19.9 & & 23.1 & 71.2 & 17.4 \\
\hline 9 & & 0.001 & & 19.3 & & 23.6 & 69.1 & 16.5 \\
\hline
\end{tabular}




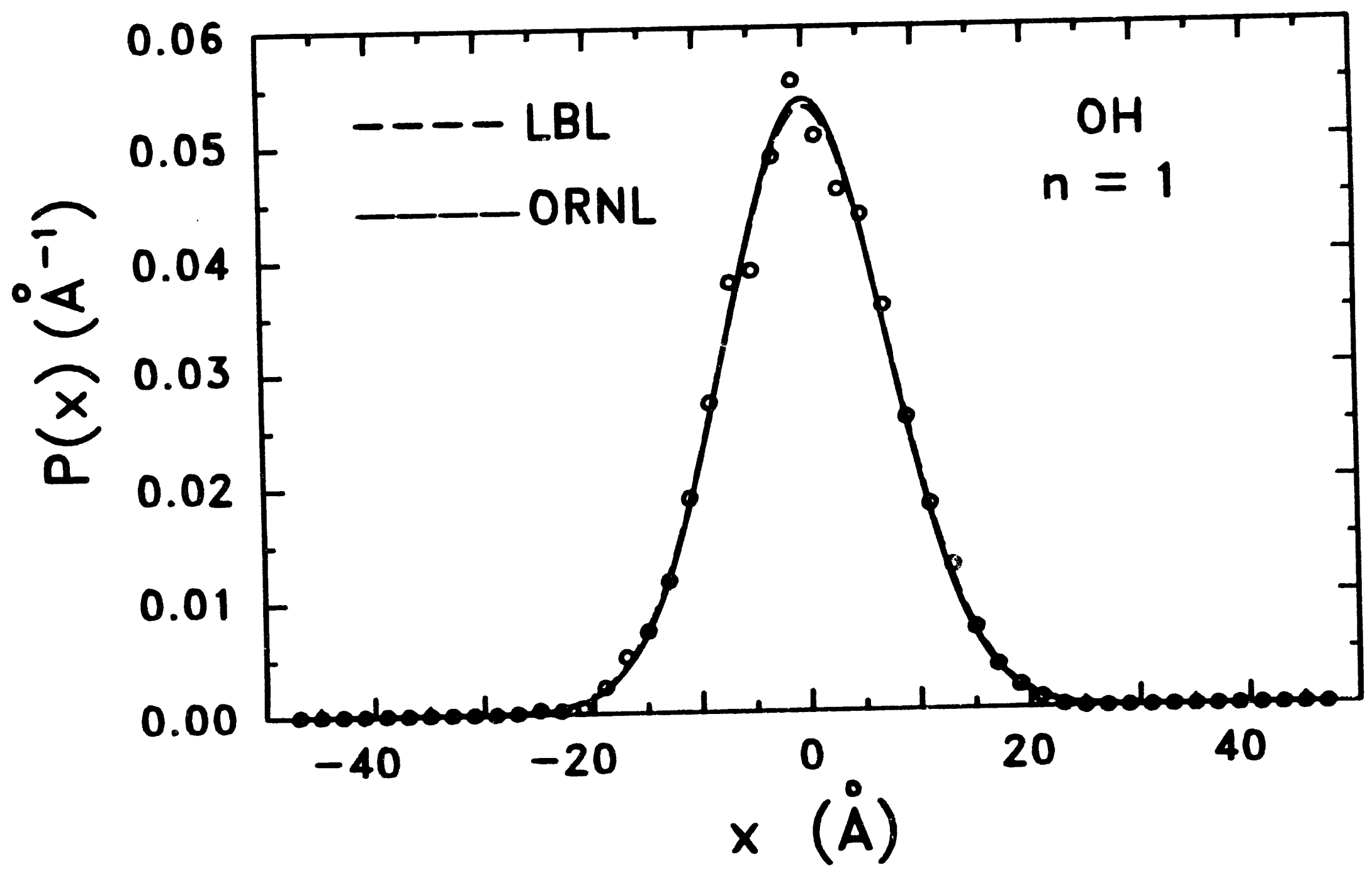




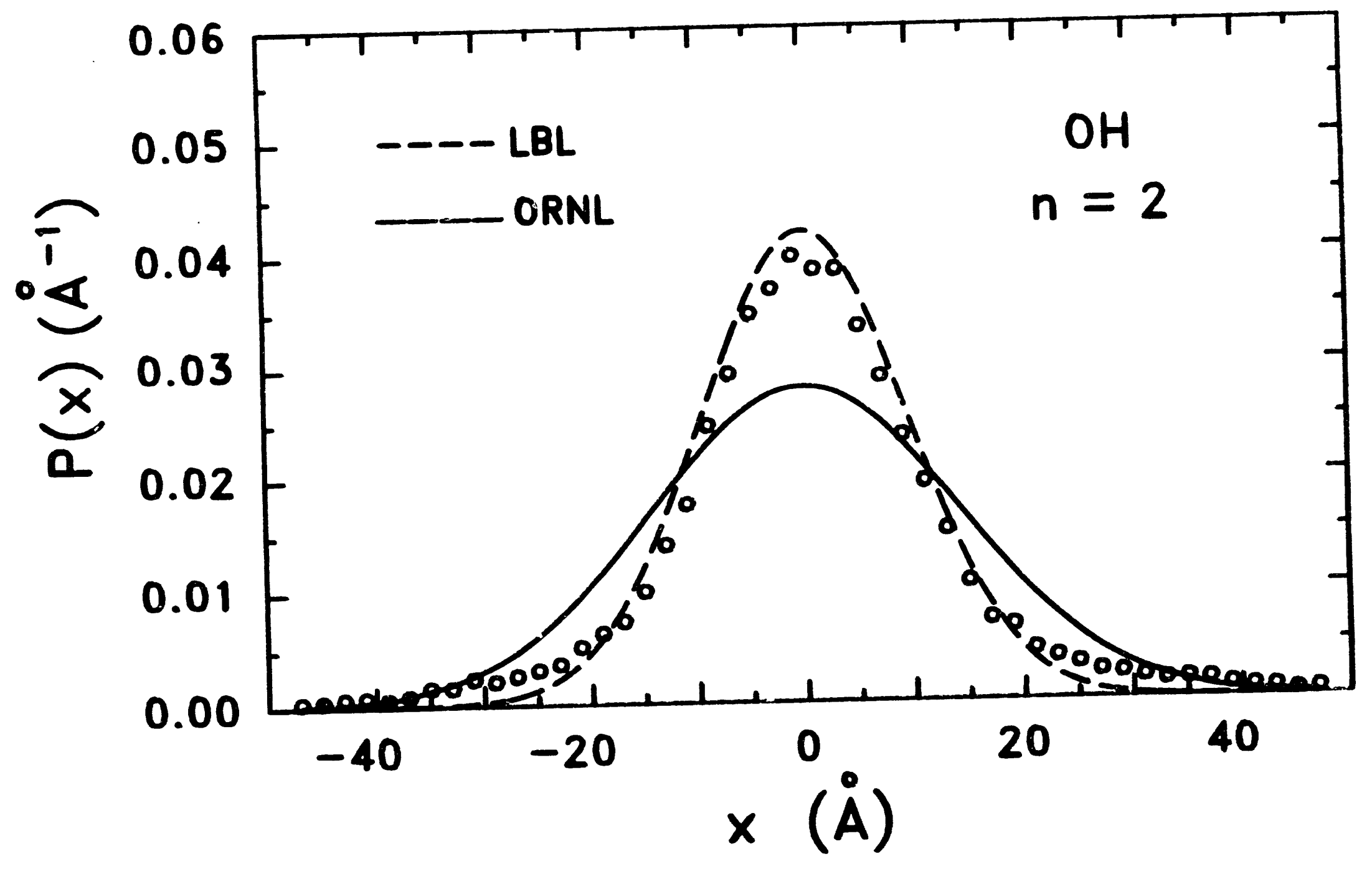




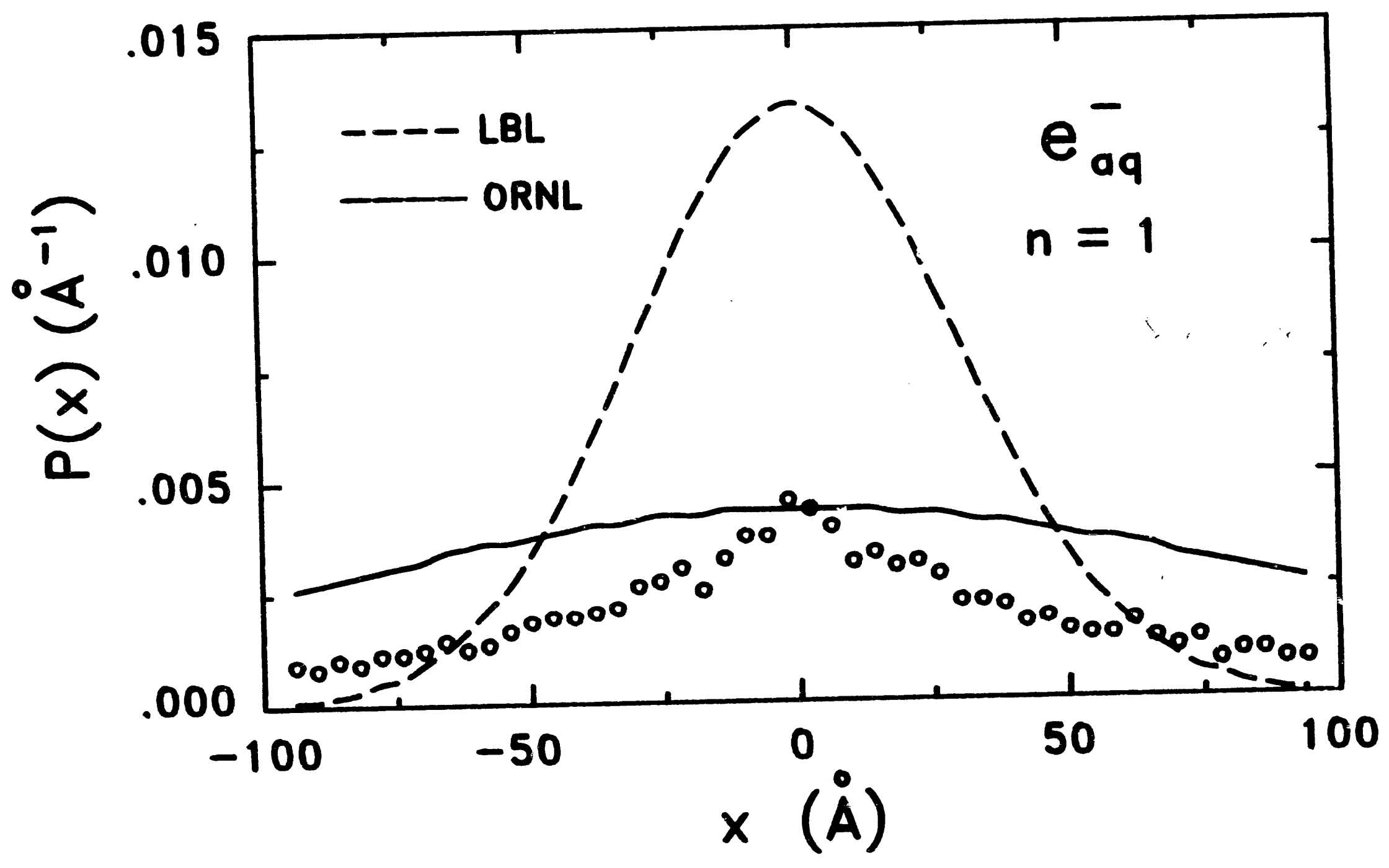




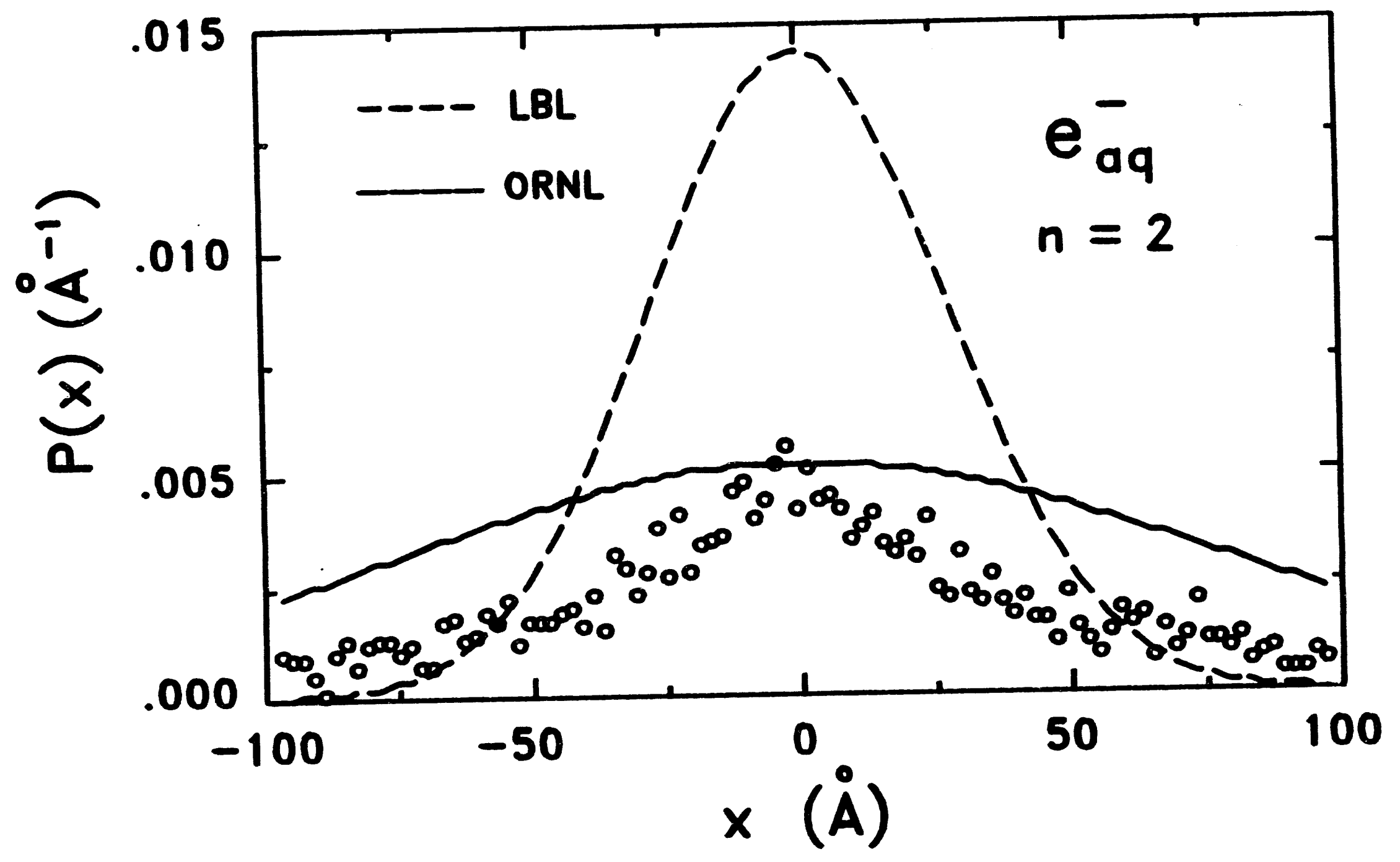




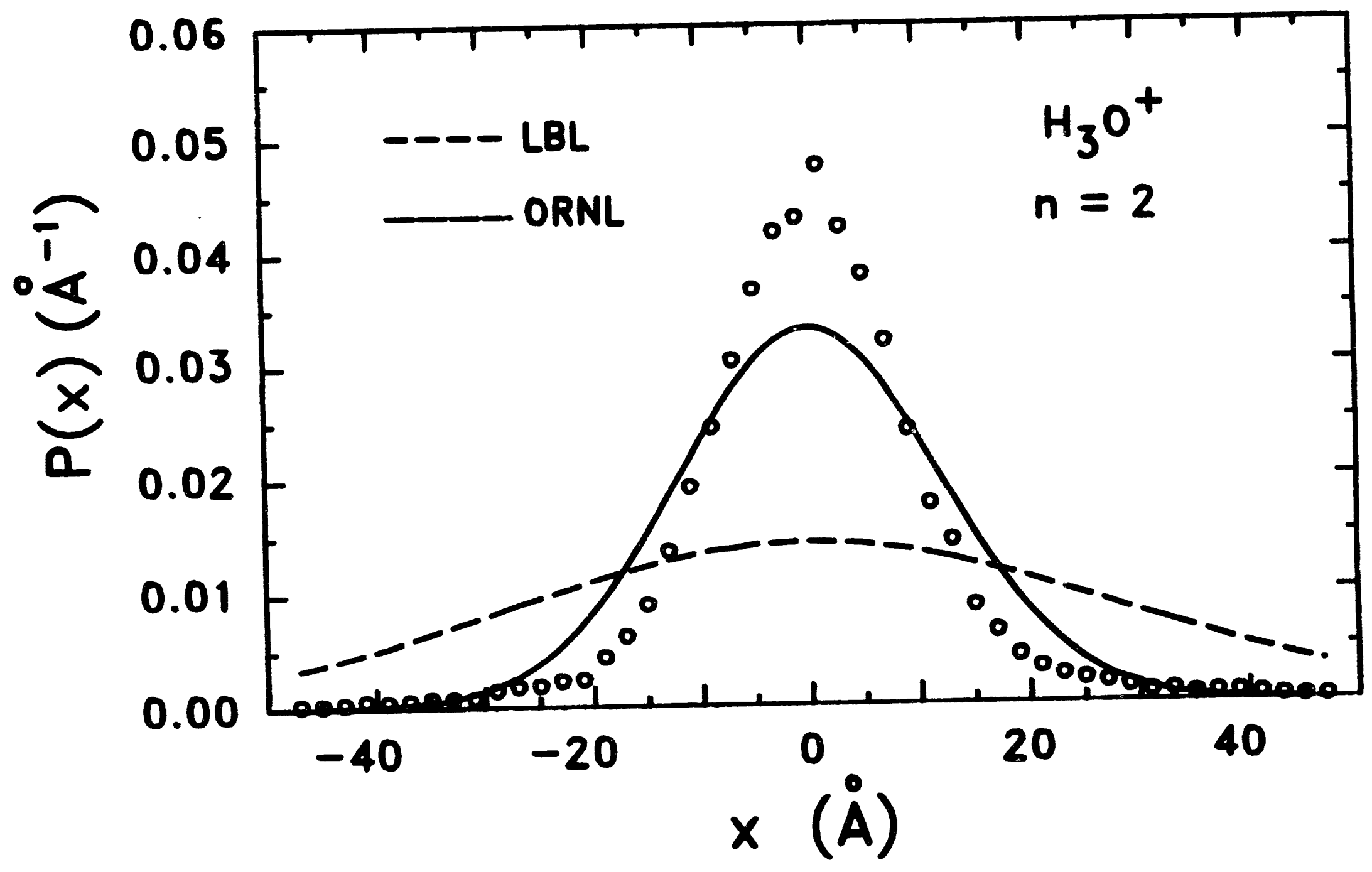



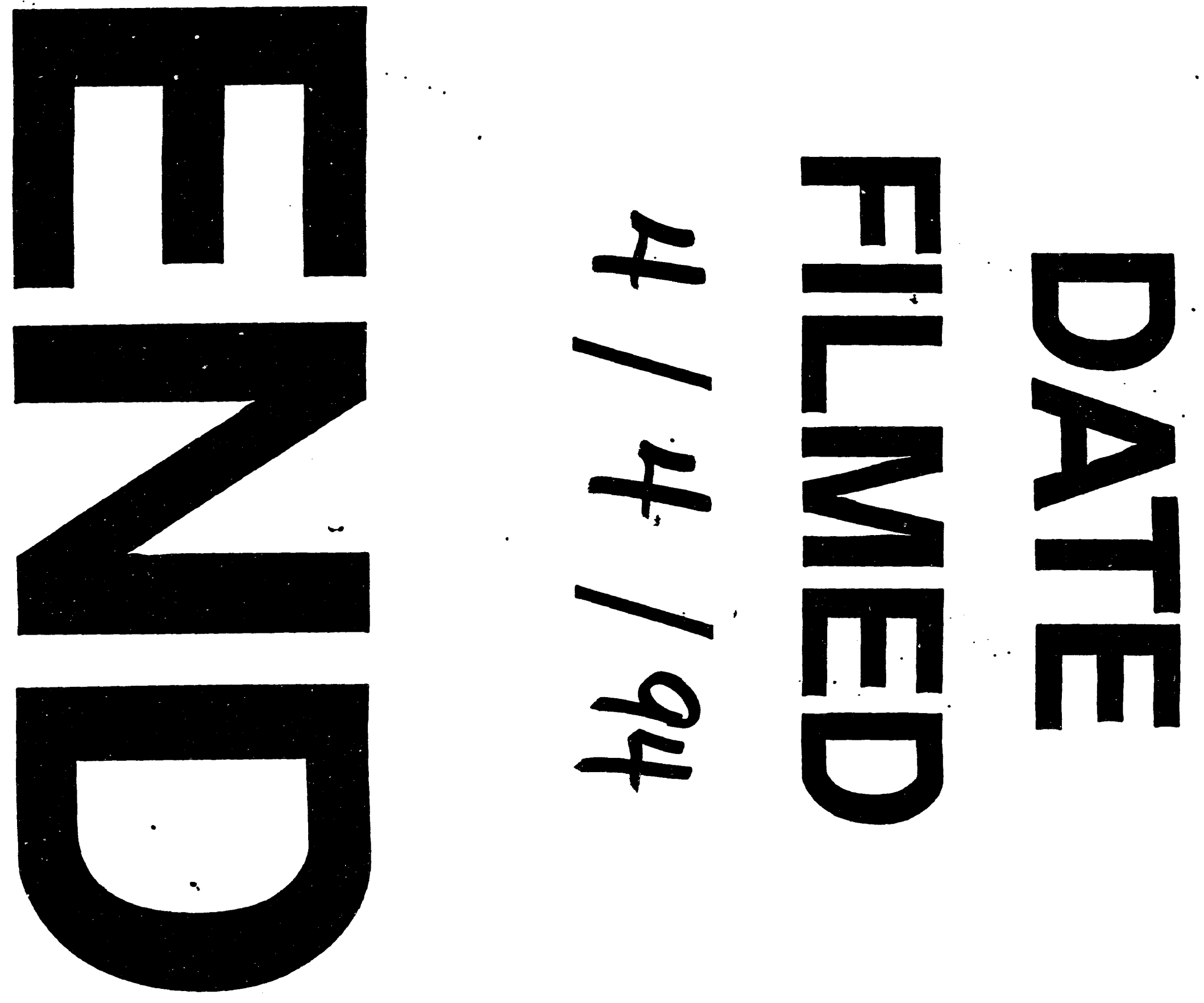
